\title{
A Multi-Objective Routing Strategy for QoS and Energy Awareness in Software-Defined Networks
}

\author{
Adriana Fernández-Fernández, Student Member, IEEE, Cristina Cervelló-Pastor, Member, IEEE \\ and Leonardo Ochoa-Aday, Student Member, IEEE
}

\begin{abstract}
Energy consumption is a key concern in the deployment and operation of current data networks, for which Software-Defined Networks (SDN) have become a promising alternative. Although several works have been proposed to improve the energy efficiency, these techniques may lead to performance degradations when QoS requirements are neglected. Inspired by this problem, this letter introduces a new routing strategy, jointly considering QoS requirements and energy awareness in SDN with in-band control traffic. To that end, we present a complete formulation of the optimization problem and implement a MultiObjective Evolutionary Algorithm. Simulation results validate the performance improvement on critical network parameters.
\end{abstract}

Index Terms-Software-Defined Networks, Energy-aware routing, Quality of Service, Multi-Objective Evolutionary Algorithm, In-band control traffic.

\section{INTRODUCTION}

$\mathbf{R}$ ECENT studies have brought the attention to the growing energy consumption of telecommunications networks. According to [1] the power consumption of the global Internet could rise to more than $10 \%$ of the world's electricity by 2025 . An effective strategy to solve this issue is to minimize the number of unused active elements, by putting them into a low-power sleep state (sleep mode) [2].

In this context, the recent architecture of Software-Defined Networks (SDN) [3] has become a potential alternative. The reason is that in this technology the logically centralized controller can flexibly manage the status of underlying forwarding devices according to the dynamically changing traffic scenario, using its global knowledge of the network state and programmable capabilities. Therefore, a routing solution considering energy efficiency as well as quality of service (QoS) requirements can be easily implemented in SDN.

Although energy-aware routing has been extensively treated, few works in SDN take QoS into consideration. For instance, the existing trade-off between energy consumption and QoSrelated parameters is discussed in the approach presented in [4]. This work simultaneously optimizes the power saving and the network performance in software defined data center networks, according to a pre-defined combination of quality requirements. Similarly, the multi-domain routing reported in [5] takes into account quality of transmission and energy saving in software-defined optical networks. These two objectives are balanced considering connection requests separated

The authors are with the Department of Network Engineering, Universitat Politècnica de Catalunya, Esteve Terradas, 7, 08860, Castelldefels, Spain, e-mails: \{adriana.fernandez, cristina, leonardo.ochoa\} @entel.upc.edu.

This work was supported by the Ministerio de Economía y Competitividad of the Spanish Government under project TEC2016-76795-C6-1-R and AEI/FEDER, UE and through a predoctoral FPI scholarship. into two classes of services. The use of a multi-objective evolutionary algorithm for this purpose has been proposed in [6] for dynamic optical networks with a centralized software defined integrated control plane, considering energy saving as the secondary objective for high priority traffic. However, in all these works dedicated links between the controller and forwarding nodes were considered, failing to extensively examine a practical implementation modality of SDN (i.e. in-band mode). Furthermore, performance indicators that are crucial in the correct operation of SDN (such as control traffic delay) need to be included in this analysis.

Different from the aforementioned works, this letter considers the in-band mode and derives a more fine-grained multiobjective (MO) routing approach, which enables the reduction of power consumption without performance degradation and includes integrated routing considerations for data and control plane traffic in SDN. More precisely, given a current network topology and the controller location, the proposed algorithm will find how data traffic demands and associated control traffic should be routed such that the energy consumption, the control traffic delay and the blocking rate are minimized. The results indicate that, according to the traffic type, the proposed model can significantly improve these metrics.

\section{Problem Formulation}

We consider a SDN modeled as a directed graph $G=(V, E, C)$, where $V, E$ and $C$ denote the set of nodes, links and controllers respectively, being $C \subset V$. We use $c_{e}$ to denote the capacity of a link $e \in E$. We define the set of interconnection devices as $S=\{n \mid n \in V \wedge n \notin C\}$.

Each incoming demand $k$, has associated its QoS requirements imposed by the Service Level Agreement (SLA), in terms of bandwidth and latency, denoted by $b_{k}$ and $l_{k}$, respectively. For the control plane communications, we use $T$ to denote the set of all combinations between controllers and switches in the network. In this respect, we will use $F_{k}$ to denote the overall set of traffic flows in the network related to demand $k$ (i.e. $k+T \subseteq F_{k}$ ). For each flow $f \in F_{k}$ we use $s_{f}$ and $d_{f}$ to denote its source and destination, respectively.

Let $P_{f}$ be the set of paths that can be used to route each flow $f \in F_{k}$, being $P_{k}$ the notation used to identify the subset of paths corresponding to demand $k$. In addition, let $P_{e}^{f} \subset P_{f}$ be the subset of paths that use link $e \in E$, for each $f \in F_{k}$ and $P_{c}^{k} \subset P_{k}$ denote the subset of data paths that pass through controller $c \in C$, which will not be used to route data plane communications. We use $b_{p}$ and $l_{p}$ to denote the minimum bandwidth and total latency of a path $p \in P_{f}$, respectively. 
Considering the entire set of demands fixed and known in advance, all the optimal control and data paths in terms of QoS requirements and energy efficiency can be computed jointly in a global optimization process. To formulate the MO optimization problem proposed, the required variables, objective functions and constraints are defined as follows:

$x_{e}$ : Binary variable that indicates whether a link $e \in E$ is active.

$\gamma_{f, p}$ : Binary variable that indicates whether a path $p \in P_{f}$ is selected to route a traffic flow $f \in F_{k}$.

$w_{f, p}$ : Cost of using a path $p \in P_{f}$ to route a traffic flow $f \in F_{k}$.

$\lambda_{i}$ : Binary variable that indicates whether a node $i \in S$ belongs to the selected data path.

Minimize the number of active links in the network:

$$
\operatorname{minimize} \sum_{e \in E} x_{e}
$$

Minimize the total cost of routing the incoming demand:

$$
\operatorname{minimize} \sum_{f \in F_{k}} \sum_{p \in P_{f}} \gamma_{f, p} w_{f, p}
$$

To route the incoming traffic demand $k$, a single data path is selected. Afterward, control messages are sent from controllers only to those switches belonging to the selected data path.

$$
\sum_{p \in P_{f}} \gamma_{f, p}= \begin{cases}1 & f=k \\ \lambda_{d_{f}} & \forall f \in T, d_{f} \in S\end{cases}
$$

Paths passing through any controller $c \in C$ cannot be used to route a data plane communication $k$.

$$
\gamma_{k, p}=0 \quad \forall p \in P_{c}^{k}, \forall c \in C
$$

A node $i \in S$ belongs to the data path selected to route the incoming demand $k$ if there is traffic in any of its incoming or outgoing edges, being $N(i)$ the set of neighbours of $i$.

$$
\lambda_{i} \geq \frac{1}{2} \sum_{j \in N(i)}\left(\sum_{\substack{p \in P_{k} \\(i, j) \in p}} \gamma_{k, p}+\sum_{\substack{p \in P_{k} \\(j, i) \in p}} \gamma_{k, p}\right) \quad \forall i \in S
$$

The cost of using a data path to route the incoming traffic demand $k$ depends on the gap between the requested amount of resource quality and the available ones. Likewise, the cost of using a control path is related with its latency, being $l_{f}^{s p}$ the shortest path delay of a control traffic flow $f \in T$.

$$
w_{f, p}= \begin{cases}\frac{1}{2}\left[V\left(\frac{b_{p}-b_{k}}{b_{p}}\right)+V\left(\frac{l_{k}-l_{p}}{l_{k}}\right)\right] & f=k, \forall p \in P_{k} \\ \frac{l_{p}-l_{f}^{s p}}{l_{p}} & \forall f \in T, \forall p \in P_{f}\end{cases}
$$

To ensure the SLA fulfillment, data paths that do not meet QoS requirements, are penalized with an infinite cost using the validation function $V(x)$.

$$
V(x)= \begin{cases}x & x \geq 0 \\ \infty & \text { otherwise }\end{cases}
$$

A link $e$ is active if it is used by some path $p \in P_{f}$ of some traffic flow $f \in F_{k}$

$$
x_{e} \geq \gamma_{f, p} \quad \forall f \in F_{k}, \forall p \in P_{e}^{f}, \forall e \in E
$$

The total traffic in each active link $e \in E$ is less than the link capacity.

$$
\sum_{f \in F_{k}} \sum_{p \in P_{e}^{f}} \gamma_{f, p} b_{f} \leq c_{e} x_{e} \quad \forall e \in E
$$

Using this model, the centralized controller can determine the optimal routes and set the required flow rules on each interconnection device before the traffic arrival. However, the use of traditional mathematical programming methods (such as linear combination of weights, goal programming and $\epsilon$ constraint methods) to solve a MO optimization problem has a number of important drawbacks [7]. Considering the high complexity of the presented MO optimization problem in current real-world networks, in the next section an effective routing scheme is proposed based on the use of evolutionary algorithms.

\section{Multi-Objective Routing Approach}

Evolutionary algorithms have been extensively used in MO problems involving multiple conflicting objectives and intractable large and highly complex search spaces [8]. These mechanisms, known as Multi-Objective Evolutionary Algorithms (MOEA), aim to find or approximate a group of tradeoff solutions called the Pareto-optimal solution set.

A solution is Pareto-optimal when no improvement can be made on one objective value without degrading any other. This allows to find several optimal solutions in a single run of the algorithm, instead of having to perform a series of separate runs as in mathematical techniques. Then a preferred solution from this set can be chosen based on user-defined criteria. Moreover, even with an incorrect initial parameter setting, they are robust enough to provide fairly good results [9].

The particular MOEA proposed in this work is based on the Strength Pareto Evolutionary Algorithm 2 (SPEA2) [10], which is a very effective approach that outperforms other MOEAs. SPEA2 implements a Pareto-based fitness assignment strategy with density estimation to determine the relevance of each individual in the current population. At each generation, the non-dominated solutions found are kept in a secondary population $(S)$, which is the outcome of the algorithm once the terminal condition is reached. The following outlines the specific considerations done to customize these basic concepts to our particular routing problem.

\section{A. Algorithm Design Issues}

The key idea of this proposal is to fully take advantage of the high control flexibility given by the dynamic configuration capabilities and centralized network view of SDN. The initially required control plane configuration (i.e. controller-switches association and node to controller control paths) is determined off-line adapting the generic integer linear problem presented in [11]. When a new demand arrives, a routing request is sent to the controller, which calculates the data and remaining control paths (from it to its associated switches), based on the proposed MO approach. The computed paths are established then, through the required flow forwarding rules. 
1) Chromosome Structure: In the proposed scheme, a candidate solution or chromosome represents a selected set of $n$ paths. The first $n-1$ routes identify the control paths used by the controller to install the flow forwarding rules in each node traversed by the data traffic. The last gene in the solution is the data path for the requested connection, being $n-1$ precisely the number of nodes along this path.

2) Initial Population: The set of solutions that form the initial population $(P)$ of an evolutionary algorithm is usually obtained by random generation. In this instance, a hybrid approach is considered. Data paths are selected randomly from the set of admissible paths, i.e. the ones that satisfy the QoS requirements. By contrast, control paths are selected considering the routes used previously by other demands, if they still have enough capacity. Hence, the traffic is likely to be concentrated on the same links, as long as it can be accommodated, reducing the number of active links. In case that no path has been already established between the controller and a node, control paths are selected randomly from the overall set of feasible routes.

3) Evaluation: To evaluate the suitability of each individual in the population, two objective functions were defined according to the conflicting goals considered in this $\mathrm{MO}$ routing strategy.

The first objective function supports performance requirements for control and data plane communications using a best-fit scheme. This behaviour is modeled in Equation (10) considering an individual $i$. Using this expression, a lower cost is assigned to individuals whose control paths $\left(p_{c}\right)$ have the closest delays to the lowest ones $\left(s p_{c}\right)$ and data path $\left(p_{d}\right)$ best satisfies the SLA thresholds. Consequently, two important routing performance metrics are improved, namely the number of future requests that can potentially be accommodated is increased and the control traffic delay is reduced. In order to avoid dominant effect, the possible values of the different parameters are normalized into the interval $(0,1)$.

$$
Q_{i}=\sum_{p_{c} \in i} \frac{l_{p_{c}}-l_{s p_{c}}}{l_{p_{c}}}+\frac{1}{2}\left(\frac{b_{p_{d}}-b_{k}}{b_{p_{d}}}+\frac{l_{k}-l_{p_{d}}}{l_{k}}\right)
$$

The second objective, related with energy awareness, aims to minimize the number of links that need to be activated when a connection request arrives. Considering $L_{i}$ as the set of links used by an individual $i$ and $A$ the record of current active links in the network, Equation (11) determines the amount of additional links required for the incoming demand.

$$
P_{i}=L_{i}-A
$$

4) Crossover: According to a crossover rate $\left(c_{r}\right)$, two solutions from the mating pool $(M)$ are selected randomly to be parents. We apply a single-point approach, where a random common node from both data paths (apart from the source and the destination node) is selected as the crossover point. Two different data paths are generated by swapping the first part and second part of both parents. Then, a loop detection process (similar to the one explained in [6]) is applied to ensure the validity of resulting data paths. Finally, the control path for each node is taken from the corresponding parent and added to form the two children.

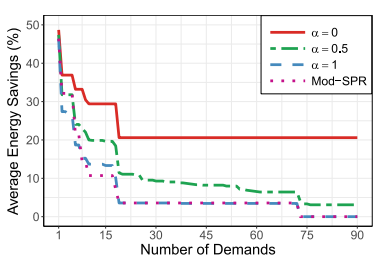

(a) Average energy saving

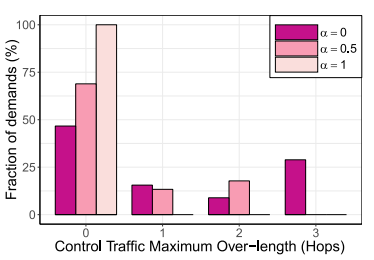

(b) Control traffic over-length.
Fig. 1. Algorithm performance in Abilene with the reported traffic matrices.

5) Mutation: Likewise, based on a mutation rate $\left(m_{r}\right)$, a random node is selected from an individual data path. A new solution is then generated considering this node as traffic source. Using this new solution, the original data path is modified from the selected node to the destination node. After applying the loop detection process, a new solution is created adding to the resulting data path, the corresponding control routes.

6) Selected solutions: To select a preferred solution we use a service-differentiation approach, i.e. the demands have been separated into two classes with different QoS requirements. Incoming traffic belonging to the class 1 (QoS_sensitive) is routed using the solution with the best performance in the first objective function. Otherwise, for demands under the class 2 (Best_effort) the solution that minimizes energy consumption is selected for transmission.

\section{RESUlTS AND Discussion}

The proposed model was tested using the Abilene topology collected from SNDlib [12], considering one node as the controller (according to the well known minimum k-median model) and the remaining nodes as SDN switches. The evolutionary parameters were empirically set to: $|P|=20,|S|=10$, $|M|=5, c_{r}=0.9$ and $m_{r}=0.1$. Two stopping criteria are considered: a) 30 consecutive iterations without improvement or b) a maximum of 100 generations.

Results have been determined with a $95 \%$ confidence interval not exceeding $4 \%$ of the indicated average values, estimated by running our algorithm 30 times with different prime number seeds on each scenario instance. Three scenarios were considered according to the $\alpha \in[0,1]$ value, which defines the proportion of demands that belongs to the class 1 , being the demands randomly allocated in one of the two classes. For the control traffic we assume an average rate of 1.7 Mbps [13].

The average running time of the algorithm, implemented in Python, takes less than $3 \mathrm{~s}$ in a computer equipped with 3.30 GHz Intel Core i7 and $16 \mathrm{~GB}$ RAM. This result is perfectly suitable for the considered use case of dynamic traffic provisioning on a large-scale transport SDN with predictable real-time connection requests, characterized by large bandwidth (due to traffic aggregation) and long durations. Furthermore, the computational complexity of the proposed MOEA is determined by the size of primary and secondary populations [10], being scalable with respect to network size.

Since there are no similar MO strategies considering inband SDN available for comparing the performance of our 


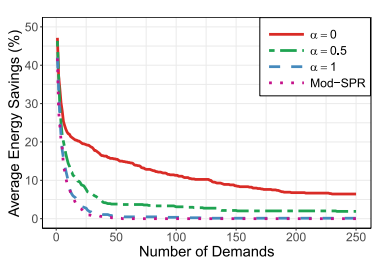

(a) Average energy saving.

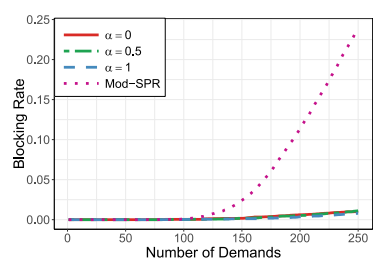

(b) Average blocking rate.
Fig. 2. Algorithm performance in Abilene with generated traffic demands.

approach, we use a modified Shortest Path Routing (ModSPR). Mod-SPR can be considered as a default shortest path routing algorithm for SDN with in-band control traffic and no data plane communications through any controller.

Fig. 1(a) shows the average energy savings with the reported traffic matrices and the number of nodes as the required delay (in terms of hops). The energy savings were computed as the number of links in sleep mode over the total amount of network links. As expected, the energy saving decreases while the number of demands grows, since new paths need to be established. It is also confirmed that less energy is saved when the amount of high-priority demands grows, due to the reduction of traffic routed through the paths minimizing the number of active links in the network. Nevertheless, even in the more demanding scenario $(\alpha=1)$ the achieved energy savings are no worse than the ones obtained with Mod-SPR.

Given the conflicting nature of the considered objectives, the best energy efficiency is achieved while sacrificing performance on QoS-related parameters. To examine this trade-off, in Fig. 1(b) we show the impact of our algorithm on control path delay, a crucial performance metric in SDN. The notation Maximum Over-length denotes the maximum difference (in number of hops) for each traffic demand between the length of the routing solution and the corresponding shortest path. As it is shown, in scenarios with larger amount of QoS_sensitive demands, the control traffic is routed using a fewer number of hops for a higher fraction of demands, avoiding performance degradations. On the contrary, when traffic is completely class $2(\alpha=0)$ the control path delay is affected in order to minimize the number of active links.

To further evaluate the implications of a more demanding traffic load in the energy efficiency and the number of accepted requests, 250 demands were generated with random sources and destinations. In order to get a higher amount of incoming requests with lower bandwidth requirements, we assume an exponentially distributed traffic rate with mean value of 100 Mbps. A random delay ranging from the shortest path length to $x$ times this value (where $x$ is the total number of switches) is used to consider a wider range of different delay requirements. It can be observed from Fig. 2(a) that a higher volume of traffic will imply the use of more active links in order to avoid the blocking and thus, less energy is saved. Although a lower number of blocked demands is expected when a higher number of demands follows the paths prioritizing the first objective function, it can be appreciated in Fig. 2(b) that the performance of our algorithm in this topology is almost the same in terms of accepted demands for the three scenarios considered. This is caused by the topological characteristics of the Abilene network, where the blocking rate depends heavily on the low redundancy of admissible data paths. More importantly, in all cases the proposed routing algorithm significantly outperforms the Mod-SPR in terms of accepted demands.

\section{CONCLUSION}

In this letter, we have proposed a multi-objective routing approach jointly considering QoS requirements and energy awareness, suitable for SDN environments with in-band control traffic. To achieve this, we have formulated an optimization problem that integrates the routing requirements for data and control traffic and implemented this approach using a MOEA based on SPEA2. Apart from being an effective routing scheme, the most significant added value of the proposal is the flexibility of driving different solutions according to the dynamically changing traffic scenario. Extensive simulations using a real topology with static and randomly generated traffic matrices validate the performance improvement on critical network parameters such as energy efficiency, control traffic delay and blocking rate. The proposed strategy is crucial and preliminary for designing online routing schemes suitable for current control planes, since it discloses and manages the intrinsic trade-off between environmental and performance concerns. As future work, we want to provide an analysis on the impact of reducing the number of active network elements on SDN reliability. Additionally, to provide optimal bounds for some limited cases will be a follow-on task.

\section{REFERENCES}

[1] R. S. Tucker, "Energy consumption in telecommunications," in Proc. 2012 Optical Interconnects Conference, May 2012, pp. 1-2.

[2] M. Gupta and S. Singh, "Greening of the Internet," in Proc. ACM SIGCOMM'03, 2003, pp. 19-26.

[3] D. Kreutz, F. M. Ramos, P. Verissimo, C. Esteve Rothenberg, S. Azodolmolky, and S. Uhlig, "Software-Defined Networking: A Comprehensive Survey," Proceedings of the IEEE, vol. 103, pp. 14-76, 2015.

[4] F. A. Moghaddam and P. Grosso, "Linear programming approaches for power savings in software-defined networks," in Proc. IEEE NetSoft Conference and Workshops (NetSoft'16), Jun. 2016, pp. 83-87.

[5] J. Zhao, Q. Yao, D. Ren, W. Li, and W. Zhao, "A Multi-Domain Control Scheme for Diffserv QoS and Energy Saving Consideration in SoftwareDefined Flexible Optical Networks," Optics Communications, vol. 341, pp. 178-187, 2015.

[6] J. Wang, X. Chen, C. Phillips, and Y. Yan, "Energy Efficiency with QoS Control in Dynamic Optical Networks with SDN Enabled Integrated Control Plane," Computer Networks, vol. 78, pp. 57-67, Feb. 2015.

[7] G. Chiandussi, M. Codegone, S. Ferrero, and F. E. Varesio, "Comparison of multi-objective optimization methodologies for engineering applications," Comput. Math. Appl., vol. 63, no. 5, pp. 912-942, Mar. 2012.

[8] C. A. Coello, G. B. Lamont, and D. A. V. Veldhuizen, Evolutionary Algorithms for Solving Multi-Objective Problems, 2007, vol. 5.

[9] H. P. Schwefel, "Advantages (and disadvantages) of evolutionary computation over other approaches," in Evolutionary Computation 1: Basic Algorithms and Operators, 2000, ch. 3, pp. 20-22.

[10] E. Zitzler, M. Laumanns, and L. Thiele, "SPEA2: Improving the Strength Pareto Evolutionary Algorithm for Multiobjective Optimization," in Proc. EUROGEN, 2002, pp. 95-100.

[11] A. Fernandez-Fernandez, C. Cervello-Pastor, and L. Ochoa-Aday, "Achieving Energy Efficiency: An Energy-Aware Approach in SDN," in Proc. IEEE GLOBECOM'16, Dec. 2016, pp. 1-7.

[12] S. Orlowski, M. Pióro, A. Tomaszewski, and R. Wessäly, "SNDlib 1.0Survivable Network Design Library," Networks, vol. 55, no. 3, pp. 276286, 2010.

[13] J. Li, J.-H. Yoo, and J. W.-K. Hong, "Dynamic control plane management for software-defined networks," International Journal of Network Management, vol. 26, no. 2, pp. 111-130, 2016. 